\title{
Time Dependent Dielectric Breakdown study of Organo Silicate Glass materials over a wide range of $\boldsymbol{k}$-values
}

\author{
Y. Barbarin, L. Zhao ', P. Verdonck, M.R. Baklanov, K. Croes, Zs. Tökei \\ Imec vzW \\ Kapeldreef 75, B-3001 Leuven, Belgium \\ ${ }^{\dagger}$ Global Foundry assignee at Imec
}

\begin{abstract}
The time dependent dielectric breakdown (TDDB) of four organo-silicate-glass (OSG) films with varying porosity $(k=2.0,2.5,2.8 \& 3.0)$ was investigated using metal-insulator-semiconductor (MIS) capacitors. Without any barrier, the dielectrics show lower TDDBlifetimes under $\mathrm{Cu}$ ion drift conditions, where the OSG2.8-film exhibits a better performance. Other results are that the damage caused by TaN/Ta barriers doesn't significantly change the TDDB performance and that the OSG-2.0-film showed excellent TDDB lifetimes.
\end{abstract}

\section{INTRODUCTION}

Scaling down the technology node requires continuous development of new low- $k$ dielectrics in back-end-ofline (BEOL) interconnects. Time dependent dielectric breakdown (TDDB) became one of the most critical reliability issues for $\mathrm{Cu} /$ low- $k$ interconnect systems $[1,2]$. For the analysis of $\mathrm{Cu}$ interconnect TDDB lifetime, different models have been proposed [3-5]. The results presented here will be plotted and compared using the conservative $E$-model. Together with the lifetime model, the breakdown mechanism is of great importance as authors have predicted dramatic reliability decrease in highly porous low- $k$ materials [6]. Such breakdown theoretically occurs either due to the weakening of the dielectric material or due to copper drift or to a combination of both. To carefully study these phenomena, TDDB studies using damascene structures are not suitable because the results can be affected by the different processing steps: low- $k$ patterning damage, $\mathrm{Cu}$ Chemical Mechanical Polishing (CMP) residues, line-edge-roughness (LER) and CD variations [7]. To access intrinsic properties of $\mathrm{Cu} /$ metal-barrier/low- $k$ structures, a planar capacitor test structure based on a metal-insulator-semiconductor (MIS) design was developed at Imec [8,9]. It was used in the current work and the TDDB performance of four OSG low- $k$ films $(\mathrm{k}=2.0,2.5,2.8$ and 3.0) were studied and compared. All films were deposited by PECVD. The 2.8 and 3.0 films do not have any sacrificial porogen, while the 2.5 and 2.0 films had porogen removed by UV assisted thermal curing. The porosity of the 2.5 and 2.0 films are respectively $25 \%$ and $40 \%$, the pore sizes are $1.5 \mathrm{~nm}$ and $3 \mathrm{~nm}$. First, the resistance to copper drift of the 2.8 and 3.0 film is studied. Then, the impact of PVD $\mathrm{TaN} / \mathrm{Ta}$ barrier deposition on the reliability of an

OSG-2.5-film [10] is investigated. Finally, we show the TDDB performance of an OSG-2.0-film with a $\mathrm{TaN} / \mathrm{Ta}$ barrier.

\section{EXPERIMENT}

The planar capacitor test structure is depicted in fig. 1 . The design allows to exclude any low- $k$ damage from etch/ash plasma, CMP and probe needle pressure. The low- $k$ films are deposited into areas of typically $100 \mu \mathrm{m} \times 100 \mu \mathrm{m}$ after the $\mathrm{SiO}_{2}$ etching step. If applicable, a physical vapor deposition PVD TaN/Ta barrier is deposited, then a $\mathrm{Cu}$ CMP process is performed and finally a standard metal passivation stack encapsulates the capacitors. In order to minimize interface effects between the Si substrate and low- $k$, a 1-nm-thick $\mathrm{SiO}_{2}$ layer is thermally grown onto the $\mathrm{Si}$ before the low- $k$ deposition. This design enables intrinsic TDDB study of barrier/low- $k$ systems and perfect area scaling was demonstrated [11].

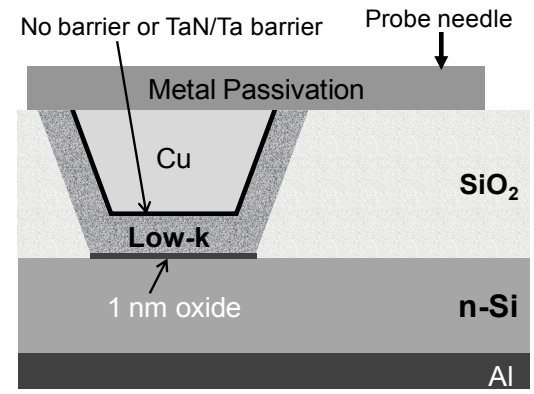

Fig. 1. Cross section schematic of MIS based capacitor structure. OSG low- $k$ films are deposited in areas of $100 \mathrm{um} \times 100 \mathrm{um}$ followed by optionally $\mathrm{TaN} / \mathrm{Ta}$ barrier deposition and conventional metallization.

(a) Positive bias +

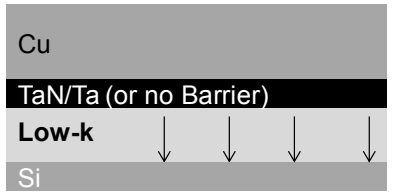

Fig. 2. Illustration of two different bias conditions. (a) $\mathrm{Cu}$ can drift into the low- $k$ when a positive bias is applied on the top electrode. (b) $C u$ is not allowed to enter into the low- $k$ when a negative bias is applied.

TDDB tests are performed under two conditions [9]. As shown in Fig. 2 a), a positive bias is applied at the top electrode so $\mathrm{Cu}$ ions can drift into the low- $k$ film under the influence of the electric field, which is the case as damascene structures. In the second configuration (Fig 2. b)), a negative bias is applied at the top electrode and $\mathrm{Cu}$ ions cannot enter into the low- $k$ dielectric. Switching the polarity, the role of $C u$ in TDDB can be 
studied by comparing the two bias conditions. TDDB tests presented below were performed at $100^{\circ} \mathrm{C}$ under various electric fields. The failure criterion was set for an abrupt change in leakage current.

\section{RESULTS AND DISCUSSION}

\section{A. OSG low-k films without any metal barrier}

First, two dense OSG films with k-values of 2.8 and 3.0 were studied without any barrier, the thickness for both films is $90 \mathrm{~nm}$. TDDB tests were performed under the same conditions. All samples had relatively tight distributions which lead to large values of the shape parameter. In fig. 3, Weibull plots of the time-to-failure (TTF) are shown for the OSG-2.8-film for (a) the positive and (b) the negative bias configurations. The shape parameter $\beta$ is 6.9 both for positive bias and negative bias. For the OSG-3.0-film (not shown here), the shape parameters $\beta$ are 6.4 for the positive bias case and 12.5 in the negative bias case.
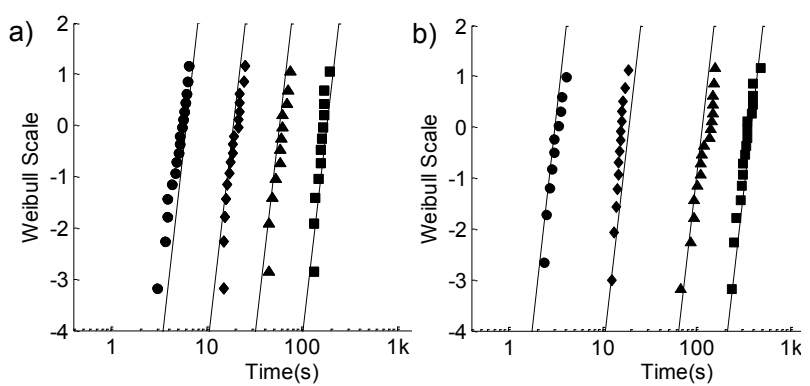

Fig. 3. Weibull plots of TDDB TTF of the OSG-2.8-film under (a) a positive bias condition and (b) a negative bias condition.

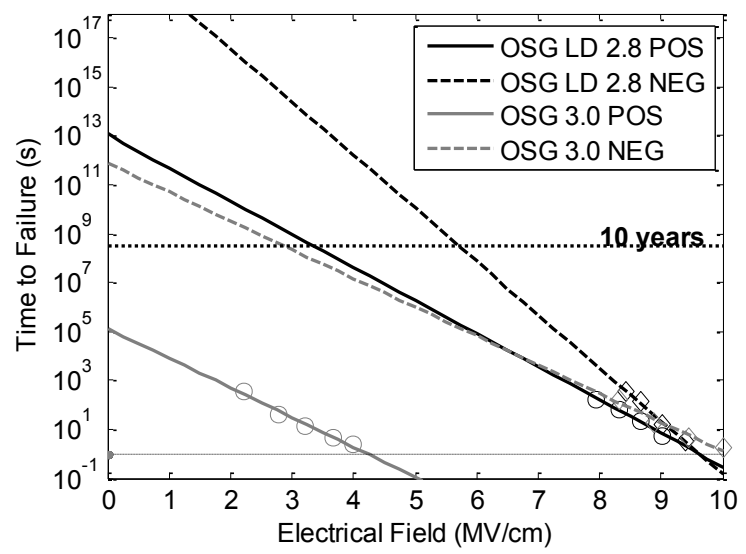

Fig. 4. TDDB $\operatorname{TTF}\left(\mathrm{t}_{63.0 \%}\right)$ vs. electric field for two OSG films with $k$-values of 2.8 and 3.0 under both positive and negative bias conditions. The fitting is done with the $E$-Model.

Fig. 4 shows the TDDB lifetimes as a function of the electric field for the two different films when applying either a positive or a negative bias. Data points were fitted using the $E$-model. Both films show large differences between negative and positive bias which means that $\mathrm{Cu}$ ions play a significant role [9]. Comparing the data with negative bias, the
OSG-2.8-film shows much higher intrinsic performance than the OSG-3.0-film with respective acceleration factors of $\gamma_{\text {neg } 2.8}=-7.0 \mathrm{~cm} / \mathrm{MV}$ and $\gamma_{\text {neg } 3.0}=-2.7 \mathrm{~cm} / \mathrm{MV}$. Under positive bias stress, the breakdown electrical field is much lower for the OSG-3.0-film which limits the TDDB lifetime below 2 days. Contrarily, the OSG-2.8-film has the same breakdown field under positive or negative bias stress. Furthermore the OSG-2.8-film meets the 10 years requirements at $1 \mathrm{MV} / \mathrm{cm}$ under positive bias without any barrier ( $\left.\gamma_{\text {pos } 2.8}=-3.1 \mathrm{~cm} / \mathrm{MV}\right)$. This indicates that $C u$ ion drift is slower into this OSG-2.8-film at $100^{\circ} \mathrm{C}$.

\section{B.OSG-2.5-film with TaN/Ta barriers}

In this section, TDDB lifetimes of OSG-2.5-films are studied for two different TaN/Ta barriers, the thickness of the barriers is the same $(\sim 6 \mathrm{~nm})$, the DC bias power during the PVD process was set to $15 \mathrm{~kW}$ and $2 \mathrm{~kW}$. A lower DC bias power is supposed to be less damaging to the low- $k$. Like in section A, TDDB data were recorded under positive and negative bias stress [9]. For completeness, a sample with no barrier was also measured in the positive bias configuration.
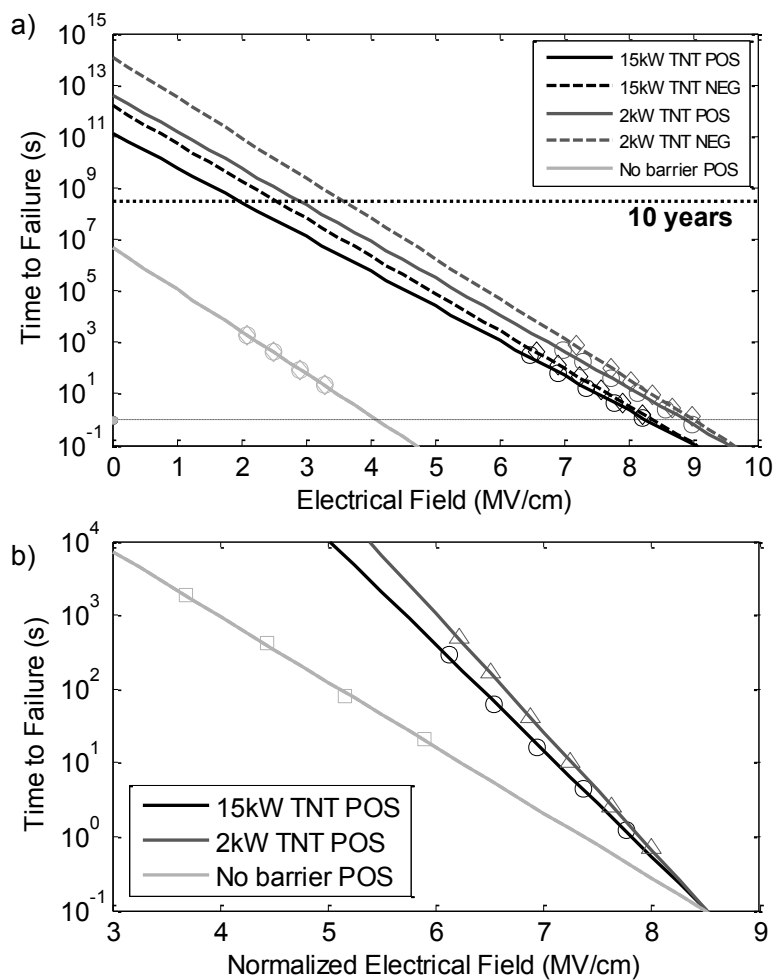

Fig. 5. TDDB TTF (t63.0\%) vs. (a) electric field and (b) vs. normalized electric field of an OSG-2.5-film for the following configuration: no barrier, $2 \mathrm{~kW} \mathrm{TaN/Ta}$ barrier, $15 \mathrm{~kW} \mathrm{TaN/Ta}$ barrier.

Analyzing the shape parameters, all four configurations recorded have tight distributions: $\beta_{\text {posNoBarrier }}=7.3$, $\beta_{\text {pos } 15 K W}=7.3, \quad \beta_{\text {pos } 15 K W}=6.8, \quad \beta_{\text {pos } 2 K W}=9.0 \quad$ and $\beta_{\text {neg } 2 \mathrm{KW}}=8.9$. TDDB times to failure for all configurations are plotted in fig. $5 \mathrm{a}$ ). The OSG-2.5 
sample without any barrier has a low breakdown field of 4.7 MV/cm. The TaN/Ta barrier samples do not show significant differences in TDDB lifetime between the positive and negative bias conditions. The only path for $\mathrm{Cu}$ to get into the low- $k$ dielectric is diffusing through the TaN/Ta barrier. Therefore, it can be concluded that $\mathrm{Cu}$ cannot penetrate through TaN/Ta barrier under TDDB stressing [9]. The low- $k$ damage is most likely induced by metal penetration and low- $k$ modification during the PVD TaN/Ta barrier deposition and hence the effective low- $k$ thickness decreases. This is illustrated by the small shift between the $2 \mathrm{~kW}$ and the $15 \mathrm{~kW}$ data in fig. 5 a). To allow for a direct comparison of the results, the film thicknesses have been normalized to a breakdown electrical field of $8.5 \mathrm{MV} / \mathrm{cm}$ in fig. $5 \mathrm{~b}$ ). The fitted acceleration factor for the $2 \mathrm{~kW} T a N / T a$ barrier is $\gamma_{\text {pos }}=-3.7 \mathrm{~cm} / \mathrm{MV}$, the acceleration factor for the $15 \mathrm{~kW}$ TaN/Ta barrier is $\gamma_{p o s}=-3.3 \mathrm{~cm} / \mathrm{MV}$ and without any barrier $\gamma_{\text {pos }}=-2.0 \mathrm{~cm} / \mathrm{MV}$. The differences in acceleration factors between the two TaN/Ta barriers with $2 \mathrm{~kW}$ and $15 \mathrm{~kW}$ DC bias power are small. This indicates that the damage to the low- $k$ caused by the deposition of a TaN/Ta barrier does not significantly change the TDDB performance.
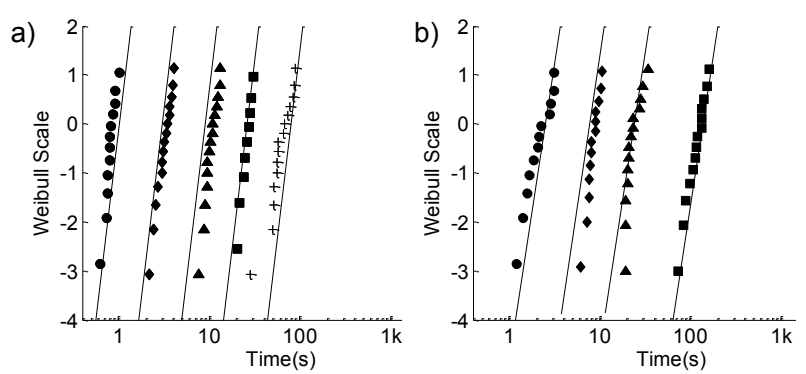

Fig. 6. Weibull plots of TDDB TTF of a 90-nm thick OSG-2.0-film under (a) a positive bias condition and (b) a negative bias condition.

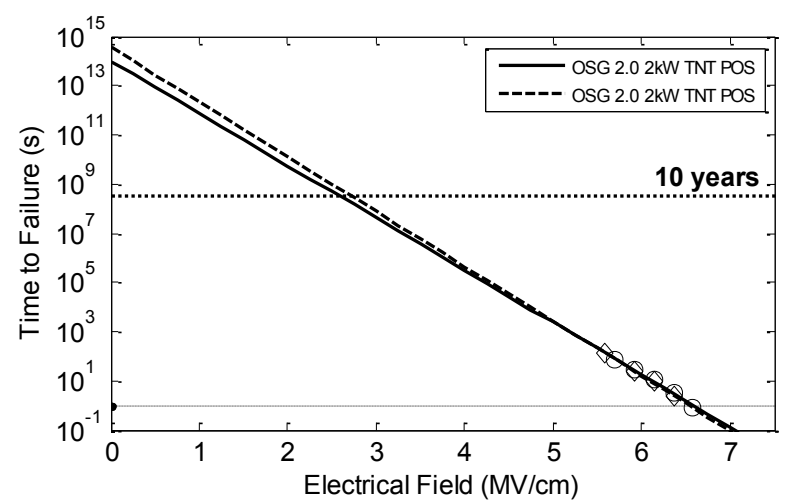

Fig. 7. TDDB $\operatorname{TTF}(\mathrm{t} 63.0 \%)$ vs. electric field of the OSG 2.0 film under both positive and negative bias conditions. (E-Model)

\section{C.OSG-2.0-film}

In this section, we present the TDDB lifetimes of our OSG-2.0-film with a TaN/Ta barrier deposited at a DC bias power of $2 \mathrm{~kW}$. The film thickness is $90 \mathrm{~nm}$. In fig. 6 , the Weibull plots of the TTF data reveal shape parameters $\beta$ of 6.7 for the positive and 5.2 for the negative bias. Fig. 7 shows the TDDB lifetimes as function of the electric field of the OSG-2.0-film. Like in section $\mathrm{B}$, the positive and negative data are on top of each other, which strongly indicates that $C u$ cannot penetrate through the TaN/Ta barrier under TDDB stressing. Compared to the other low-k films presented in sections $\mathrm{A}$ and $\mathrm{B}$, the breakdown field is only 7.0 MV/cm, which is linked to dielectric weakness due to porosity scaling [12]. However this OSG-2.0-film meets easily the 10 years requirements at $1 \mathrm{MV} / \mathrm{cm}$. The fitted acceleration factors are $\gamma_{p o s}=-4.9 \mathrm{~cm} / \mathrm{MV}$ and $\gamma_{\text {neg }}=-5.1 \mathrm{~cm} / \mathrm{MV}$. The $k$-value measurements show values of $\sim 2.5$ which indicates that this low-power $\mathrm{TaN} / \mathrm{Ta}$ barrier is still damaging, however reliable measurements are possible.

\section{CONCLUSION}

TDDB tests under positive and negative bias conditions were performed on MIS capacitors to study the reliability of four OSG films with $k$-values of 2.0, 2.5, 2.8 and 3.0. The positive bias allows $C u$ ions to potentially drift into the low- $k$, while the negative bias prevents $C u$ ions from entering the dielectric, allowing intrinsic reliability determination. Without any barrier, all low- $k$ films suffer from $C u$ ions drifts although the dense OSG-2.8-film showed better performance. Two types of $\mathrm{TaN} / \mathrm{Ta}$ layers as $\mathrm{Cu}$ diffusion barrier were studied with PVD DC bias powers of $15 \mathrm{~kW}$ and $2 \mathrm{~kW}$. Since no difference in TDDB performance was observed between the positive and negative stress conditions, we can conclude that $\mathrm{Cu}$ cannot penetrate the $\mathrm{TaN} / \mathrm{Ta}$ barriers. In addition, the damage to the low- $k$ caused by the deposition of $T a N / T a$ barriers is slightly greater at $15 \mathrm{~kW}$ DC bias power but it does not significantly change the TDDB performance of the OSG-2.5-film. Even if the $2 \mathrm{~kW} \mathrm{TaN/Ta}$ barrier damaged the top of the OSG-2.0-film, excellent TDDB lifetimes have been demonstrated.

\section{REFERENCES}

[1] Zs. Tökei et al., J. Microele. Eng. 87(3), p.384, (2009)

[2] F. Chen el al., IEEE Trans. on Elec. Dev., 56(1), p. 2, (2009)

[3] F. Chen et al., Proc. IRPS, p.46, (2006)

[4] K. Croes et al., Proc. IRPS, p.543, (2010)

[5] L. Zhao et al., J. Appl. Phys. Lett. 98032107 (2011)

[6] S.C. Lee et al. Proc. IRPS, p.2F.5.1 (2011)

[7] K. Croes et al., Proc. IRPS, p.142, (2011)

[8] L. Zhao et al., Proc. IRPS, p.848, (2009)

[9] L. Zhao et al., J. Appl. Phys. 99, 222110, (2011)

[10] L. Zhao et al., Proc. IEEE IITC, p.206, (2009)

[11] G.G. Gischia et al. Proc. IRPS, p.549, (2010)

[12] E. Van Besien et al., J. Microele. Eng. http://dx.doi.org/10.1016/j.mee.2011.04.015, (2011) 